\title{
Localisation Originale Du Lupus Tuberculeux Au Niveau De La Main
}

\author{
El Mekkaoui MJ", Kharmaz M, Lamrani MO, Mahfoud M, El Bardouni A, Berrada MS
}

Service de Traumatologie-Orthopédie, CHU Avicenne, Rabat, Morocco

DOI: $10.36347 /$ sjmcr.2020.v08i04.018

| Received: 19.03.2020 | Accepted: 27.03.2020 | Published: 27.04.2020

*Corresponding author: Mohamed Jalal EL Mekkaoui

\section{Abstract}

Cutaneous tuberculosis accounts for $2 \%$ of all extrapulmonary tuberculosis and is one of the multiple clinical presentations of extrapulmonary tuberculosis. Tuberculous lupus is a paucibacillary form of cutaneous tuberculosis in which the existence of another site of infection is very fickle. We report an original case of tuberculous lupus of the hand with the indentification of Mycobacterium tuberculosis (MT) on the skin biopsy in a 35-year-old woman. The Mantoux test was positive. The search for Mycobacterium tuberculosis in sputum was negative. The skin lesions responded to antituberculous treatment.

Keywords: Tuberculous lupus; Caseum necrosis; Mycobacterium tuberculosis.

Copyright @ 2020: This is an open-access article distributed under the terms of the Creative Commons Attribution license which permits unrestricted use, distribution, and reproduction in any medium for non-commercial use (NonCommercial, or CC-BY-NC) provided the original author and source are credited.

\section{INTRODUCTION}

La tuberculose est une pathologie qui sévit à l'état d'endémie dans les pays en voie de développement. Dans les pays industrialisés, une diminution considérable de son incidence a été observée malgré l'augmentation actuelle des cas de tuberculose dans les grandes métropoles [1].

La localisation cutanée de la maladie demeure cependant très rare et beaucoup moins fréquente que les autres localisations dont la principale est pulmonaire. Elle représente $2 \%$ des localisations extrapulmonaires de tuberculose [2]. Le diagnostic de la tuberculose cutanée est souvent difficile en raison du polymorphisme des tableaux anatomocliniques et de la multiplicité des diagnostics différentiels. L'expression clinique est déterminée par le mode d'envahissement de la peau par le bacille de Koch (BK), la virulence du germe et le degré d'immunité de l'hôte. Le lupus tuberculeux, constitue la forme clinique la plus fréquente de la tuberculose cutanée [3]. Il survient de façon lente et progressive chez des individus antérieurement contaminés par le BK et présentant une immunité modérée ou au contraire très forte contre ce bacille $[4,5]$.

Le terme de lupus a été adopté par analogie avec le loup « qui ronge les chairs ».

\section{ObServation}

Il s'agissait d'une femme âgée de 35 ans, sans antécédents pathologiques particuliers, femme de ménage de profession, consultait pour des lésions cutanées de la main gauche évoluant depuis environ un an. La patiente avait consulté 3 mois auparavant chez un autre médecin et avait été mise sous antibiothérapie anti-staphylococcique sans amélioration.

A l'examen clinique, on notait une lésion érythémateuse, rougeâtre et finement squameuse sur la face radiale du quatrième doit gauche (Fig.1). Il n'y avait pas d'adénopathie périphérique, l'état général était conservé et le reste de l'examen clinique était normale.

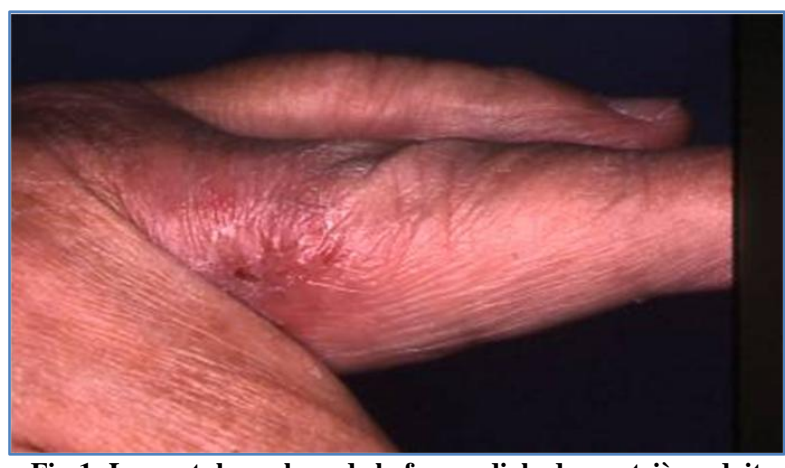

Fig-1: Lupus tuberculeux de la face radiale du quatrième doit gauche

Une biopsie cutanée concluait à une dermite granulomateuse non nécrosante. La culture du prélèvement sur milieu de Lowenstein-Jensen révélait 
du Mycobacterium tuberculosis (Fig.2). L'IDR à la tuberculine était positive à $16 \mathrm{~mm}$. La recherche des Mycobacteriums tuberculosis dans les crachats était négative.

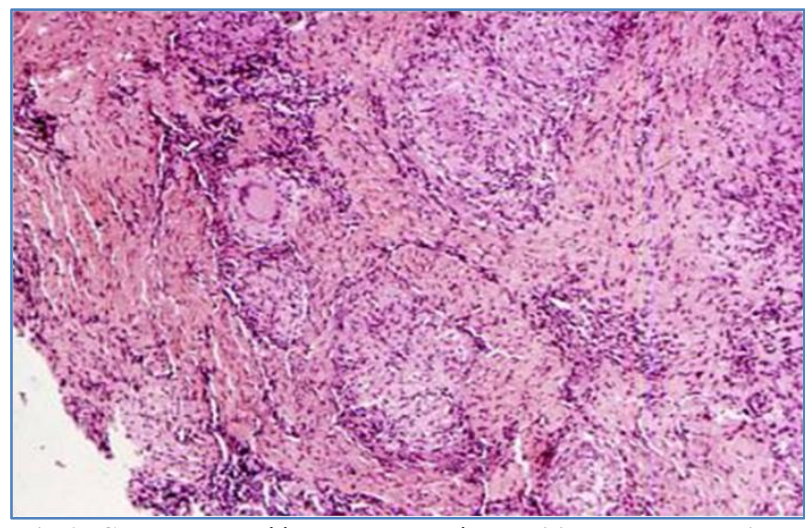

Fig-2: Culture du prélèvement cutané sur milieu de LowensteinJensen révélant du Mycobacterium tuberculosis

L'interogatoire retrouvait une notion de vaccination par le BCG. L'examen des sujets contacts n'avait révélé aucun cas de tuberculose dans l'entourage.

La patiente était traitée par trithérapie pendant 2 mois (la phase initiale) à base d'isoniazide, de rifampicine et de pyrazinamide puis 4 mois de bithérapie à base d'isoniazide et de rifampicine. L'évolution était favorable en quelque mois avec disparition des lésions cutanées.

\section{DisCUSSION}

Le lupus tuberculeux atteint deux fois sur trois la femme [2] et se présente sous des aspects cliniques très variés, parfois trompeurs. Son siège de prédilection est le visage, en particulier le nez et les joues, plus rarement le tronc et les extrémités des membres. La lésion est souvent unique, les formes multiples sont rares. Quels que soient la forme clinique qu'il emprunte et son siège, la lésion élémentaire reste toujours le lupome [4] qui est un micronodule dermique assez mou, brun jaunâtre à la vitropression (lupoïde). Le lupus tuberculeux ne s'accompagne habituellement pas de signes généraux.

$\mathrm{Au}$ cours du lupus tuberculeux, le bacille accède à la peau par voie sanguine ou par contiguïté succédant à une tuberculose sous-jacente, ganglionnaire, osseuse ou épididymaire. Rarement, il se développe sur le siège d'un chancre d'inoculation ou d'une vaccination par le BCG [5].

Le diagnostic positif repose, au cours du lupus tuberculeux, sur l'existence d'un faisceau d'arguments [6]:

- l'examen clinique qui recherchera la lésion élémentaire «le lupome »;

- la provenance d'une zone d'endémie tuberculeuse ;
- l'existence d'antécédents de tuberculose ;

- l'association à une tuberculose viscérale ou à un foyer de tuberculose viscérale active constitue un des éléments d'orientation. Elle est cependant rare et n'est retrouvée que dans 10 à $20 \%$ des cas au cours du lupus tuberculeux ;

- une réaction tuberculinique fortement positive, voire phlycténulaires témoignant de la bonne immunité du terrain.

Le traitement de la tuberculose cutanée isolée ou associée à une autre localisation est identique au traitement de la tuberculose pulmonaire. Il comporte, en phase initiale, une tri- ou une quadrithérapie afin d'éviter l'acquisition de résistances et pour rendre le patient rapidement non contagieux. Le traitement doit comporter les deux premiers mois l'isoniazide à la dose de 3 à $5 \mathrm{mg} / \mathrm{kg}$ par jour, la rifampicine $(10 \mathrm{mg} / \mathrm{kg}$ par jour), l'éthambutol (15 à $20 \mathrm{mg} / \mathrm{kg}$ par jour) et le pyrazinamide (20 à $30 \mathrm{mg} / \mathrm{kg}$ par jour), simplifiés pour les quatre mois suivants par une bithérapie isoniazide et rifampicine $[7,8]$.

La réponse au traitement spécifique antituberculeux peut constituer la seule preuve pour le diagnostic d'une tuberculose cutanée [6-8].

L'évolution du lupus tuberculeux, autrefois inexorablement mutilante, bien que lente sur de nombreuses années, a été transformée par le traitement antituberculeux qui n'empêche pas la persistance d'une cicatrice indélébile. Les complications sont devenues rares, celles liées à la surinfection à pyogènes survenant sur des lupus ulcérés ont aujourd'hui disparu. Les lupus des membres peuvent se compliquer de lymphoedème et d'éléphantiasis. Des carcinomes spinocellulaires peuvent compliquer des lupus anciens, surtout s'ils ont été traités par radiothérapie $[9,10]$.

Notre patiente avait un lupus tuberculeux avec mise en évidence du MT sur le prélèvement cutané. Dans la littérature, le MT est rarement mis en évidence et le diagnostic de lupus tuberculeux est retenu sur les arguments anamnestiques et anatomocliniques.

\section{CONCLUSION}

Il s'agit d'un cas original de lupus tuberculeux de la main avec isolement de MT permettant de confirmer le diagnostic. La guérison a été obtenue sous traitement antituberculeux.

\section{REFERENCES}

1. Ebelin M, Roulot E. Infections de la main et des doigts. Rev de Rhumatisme. 2001; 68: 520529.

2. Fenniche S, Ben Jennet S, Marrak H, Khayat O, Zghal M, Ben Ayed M. Tuberculose cutanée: aspect anatomopath et évolutif (26 cas). Ann Dermatol Venereol. 2003; 130: 1021-4. 
3. Bhutto AM, Solangi A, Khaskhely NM, Arakaki H, Nonaka S. Clinical and epidemiological observations of cutaneous tuberculosis in Larkana Pakistan. Int J Dermatol. 2002; 41: 159-65.

4. Zahaf A, Turki H, Masmoudi A. Lupus tuberculeux. Ann Dermatol Venereol. 2004; 131 : 503-7.

5. El Fekih N, Fazaa B, Kerkeni N, Sfia M, Zeglaoui F, Zermani R, Bouacha H, Kamoun M.R. Le lupus tuberculeux. Médecine et Maladies Inféctieuses. 2009; 39: 409-412.

6. Lipsker D, Grosshans E. What is lupus vulgaris in Dermatology? Ann Dermatol Venereol. 2005; 211: 189-90.
7. Sehgal VN, Sardana K, Sharma S. Inadequacy of clinical and/or laboratory criteria for the diagnosis of lupus vulgaris. J Dermatol Treat. 2007; 11: 1-4.

8. Akoglu G, Karaduman A, Boztepe G, Ozkaya O, Sahin S, Erkin G. A case of lupus vulgaris successfully treated with antituberculous therapy despite negative PCR and culture. Dermatology. 2005; 21: 290-2.

9. Yerushalmi J, Grunwald MH, Halevy DH, Avinoach I, Halevy S. Lupus vulgaris complicated by metastatic squamous cell carcinoma. Int $J$ Dermatol. 1998; 37: 934-5.

10. Zahaf A, Turki H, Masmoudi A. Lupus tuberculeux. Ann Dermatol Venereol. 2004; 131 503-7. 